\title{
Investigação da resistência à compressão e da resistividade elétrica de concretos com diferentes tipos de cimento
}

\author{
R. A. Medeiros-Junior ${ }^{1}$, M. G. Lima1, M. H. F. Medeiros ${ }^{2}$, L. V. Real ${ }^{2}$ \\ ${ }^{1}$ Departamento de Engenharia Civil, Instituto Tecnológico de Aeronáutica (ITA), Brasil. \\ ${ }^{2}$ Departamento de Construção Civil, Universidade Federal do Paraná (UFPR), Brasil.
}

\section{Información del artículo DOI: \\ http://dx.doi.org/10.21041/ra.v4 $\underline{i 2.21}$ \\ Artículo recibido el 12 de febrero de 2014, revisado bajo las políticas de publicación de la Revista ALCONPAT y aceptado el 02 de mayo de 2014. Cualquier discusión, incluyendo la réplica de los autores, se publicará en el primer número del año 2015 siempre y cuando la información se reciba antes del cierre del tercer número del año 2014.}

(C) 2014 ALCONPAT Internacional

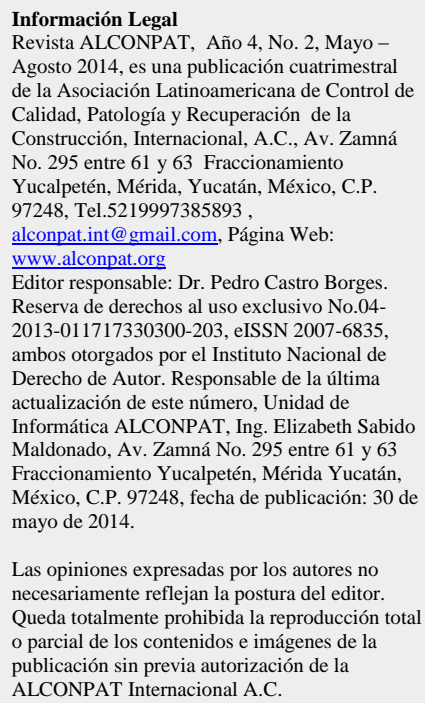

\section{RESUMO}

Nesse estudo foi possível observar a influência de quatro tipos de cimento brasileiros na resistência à compressão e na Resistividade Elétrica Superficial (RES) de amostras de concreto. Foram analisadas três relações água/cimento, o que resultou em doze distintas dosagens. Os resultados mostraram que os tipos de cimentos tem influência direta em ambos os ensaios. De maneira geral, quando comparado com uma série de referência, os cimentos com adição de escória de alto forno e pozolanas apresentaram redução na resistência à compressão do concreto, porém ganho na RES. O cimento com adição de pozolana é o que apresenta o maior ganho de resistência à compressão no tempo, embora tenha o menor valor absoluto. Os resultados também indicaram que a RES cresce com o tempo de ensaio e diminui com o aumento da relação a/c do concreto. Foi encontrada uma boa correlação entre os ensaios, com $\mathrm{R}^{2}$ variando de 0,823 a 0,999 .

Palavras chaves: resistência à compressão; resistividade elétrica superficial; cimentos; escória de alto forno; pozolana.

\section{ABSTRACT}

This work studied the influence of four Brazilian types of cement on the compressive strength and electrical resistivity in samples of concrete. Three water/binder ratios were analyzed, which led to the preparation of twelve different samples. The results show that the types of cement has a direct influence on both tests. In general, compared to a reference, the cements with additions of blast furnace slag and pozzolans reduced the strength, but increased the electrical resistivity. It was also observed that the cement with pozzolan showed the highest gain in strength with time, although it resulted in the lowest absolute value. The results also indicated that the electrical resistivity increases with time and decreases with higher water/binder ratios. A good correlation was found between the mentioned tests with R2 ranging from 0.823 to 0.999 .

Keywords: compressive strength; electrical resistivity; cements; blast fumace slag; pozzolan.

Autor de contacto: Ronaldo A. de Medeiros-Junior (ronaldodemedeirosjr@ yahoo.com.br) 
128Revista ALCONPAT, Volumen 4, Número 2, Mayo - Agosto 2014, Páginas 113 - 128

\section{INTRODUÇÃO}

O ensaio de resistência à compressão é bastante utilizado na engenharia para controle de obra e verificação do dimensionamento do concreto. A Norma Brasileira (NBR 12655, 2006), por exemplo, estabelece como critério para controle de aceitação do concreto os ensaios de resistência à compressão e de consistência (abatimento do tronco de cone). Sabe-se que alguns fatores interferem nessa propriedade do concreto endurecido, como a quantidade de água, o tipo de agregado, a condição de cura e a idade da amostra (Silva, 2009; Helene, 2011; Solís et al, 2012; Medeiros et al, 2012; Gayarre et al, 2014). No Brasil, é comum a utilização de diferentes tipos de cimentos nas construções e, devido a inclusão de adições minerais na própria fabricação, esses cimentos apresentam composições químicas e físicas distintas entre sí, resultando em diferentes propriedades do concreto, inclusive com alterações na resistência mecânica.

Apesar da importância desse ensaio, a necessidade de inserir o fator durabilidade no dimensionamento tem estimulado o desenvolvimento de novos modelos (Andrade, 2004) para a predição da vida útil de estruturas de concreto. Para isso, o uso de dados provenientes de ensaios não destrutivos é incentivado. Sabe-se que a resistividade elétrica é um parâmetro relacionado com a permeabilidade, e consequentemente, com a resistência do concreto à penetração de agentes agressivos, tais como o dióxido de carbono e os cloretos (Andrade e D’Andrea, 2011).

A Resistividade Elétrica Superficial (RES) pode ser monitorada no tempo através de métodos de ensaios não destrutivos, de baixo custo e de fácil execução. O método dos quatro pontos, também conhecido como método de Wenner, consiste na técnica mais conhecida e empregada para a obtenção da RES do concreto (Andrade e D’Andrea, 2011; Lübeck et al, 2012). Trata-se de um parâmetro interessante para uso na modelagem de vida útil de estruturas de concreto, principalmente devido à praticidade de execução. A característica não destrutiva do ensaio permite a sua contínua reprodução para monitoramento da qualidade do concreto, sem comprometer a integridade física da estrutura.

Diversos estudos (Alonso et al, 1988; Andrade et al, 1996; Gulikers, 2005; Ghods et al, 2007) consideram a RES como um fator importante que afeta a velocidade ou taxa de corrosão das armaduras do concreto.

A resistividade indica a habilidade do material em transportar cargas elétricas. Esse parâmetro é definido como o inverso da condutividade (Halliday e Resnick, 1994). Portanto, é de se esperar que cimentos com adições diferentes em sua composição influenciem nas medidas de RES.

Sendo assim, o objetivo deste estudo foi analisar a influência de quatro tipos de cimento brasileiros na resistência à compressão e na RES de amostras de concreto, buscando uma correlação entre esses ensaios.

Outros autores (Santor et al, 2012) realizaram ensaios de resistividade elétrica em amostras de concreto com diferentes tipos cimento, porém com a intenção de avaliar a influência de materiais de proteção. Em suas análises, esses autores não utilizaram cimento com escória de alto forno e ficou claro nos resultados que o tipo de cimento altera a resistividade elétrica do concreto.

Alguns outros estudos (Pereira, 2001; Tessari, 2001; Crauss, 2010) também já verificaram variações na resistência à compressão do concreto de acordo com o tipo de cimento utilizado. Porém, não foram encontrados trabalhos que avaliem a influência de uma variedade considerável de tipos diferentes de cimentos, mantendo-se todos os outros parâmetros do concreto constantes, inclusive sem a utilização de aditivos químicos, como o proposto pelo presente artigo. 
Revista ALCONPAT, Volumen 4, Número 2, Mayo - Agosto 2014, Páginas 113 - 128

\section{PROGRAMA EXPERIMENTAL}

Foram moldados corpos de prova cilíndricos (100 mm de diâmetro por $200 \mathrm{~mm}$ de altura) e cúbicos (arestas de $250 \mathrm{~mm}$ ) para realização dos ensaios de resistência à compressão e Resistividade Elétrica Superficial (RES), respectivamente. A preparação dos corpos de prova utilizados no ensaio de resistência à compressão foi realizada conforme Normas Brasileiras (NBR 5738, 2003; NBR 5739, 2007). A escolha da dimensão dos corpos de prova utilizados no ensaio de RES foi baseada nas recomendações de outro estudo (Gowers e Millard, 1999), que leva em consideração as dimensões mínimas necessárias para que as linhas de correntes geradas pelo equipamento no interior do concreto se fechem e, dessa forma, não ocasionem alterações nos valores obtidos durante o ensaio. Os materiais e as proporções utilizadas são apresentados na Tabela 1.

Tabela 1. Dosagens das amostras de concreto investigadas.

\begin{tabular}{|c|c|c|c|c|}
\hline Cimento & Cimento (kg) & Areia (kg) & Brita (kg) & Água (kg) \\
\hline CP II-F 32 & 420 & 588 & 882 & 168 \\
\hline CP II-F 32 & 420 & 588 & 882 & 210 \\
\hline CP II-F 32 & 420 & 588 & 882 & 252 \\
\hline CP III 40 RS & 420 & 588 & 882 & 168 \\
\hline CP III 40 RS & 420 & 588 & 882 & 210 \\
\hline CP III 40 RS & 420 & 588 & 882 & 252 \\
\hline CP IV 32 & 420 & 588 & 882 & 168 \\
\hline CP IV 32 & 420 & 588 & 882 & 210 \\
\hline CP IV 32 & 420 & 588 & 882 & 252 \\
\hline CP V ARI & 420 & 588 & 882 & 168 \\
\hline CP V ARI & 420 & 588 & 882 & 210 \\
\hline CP V ARI & 420 & 588 & 882 & 252 \\
\hline
\end{tabular}

É possível observar na Tabela 1 que foram moldados corpos de prova para doze dosagens distintas de concreto, que diferem entre si pela quantidade de água (relações a/c de $0,4,0,5$ e 0,6 ) e pelo tipo de cimento (4 tipos). Todos as outras proporções foram mantidas constantes entre as diferentes dosagens investigadas. O tamanho máximo do agregado foi de $9,5 \mathrm{~mm}$. Não foram utilizados aditivos químicos em nenhuma das amostras de concreto, por se acreditar que este tipo de material pode introduzir mudanças na resistividade elétrica do compósito cimentício.

Quatro tipos de cimento disponíveis comercialmente no Brasil foram utilizados. Esses materiais possuem composições químicas diferentes devido à presença ou não de adições. As seguintes Normas Brasileiras regem a especificação dos cimentos utilizados neste estudo: (a) CP II-F 32 cimento Portland composto - especificação (NBR 11578, 1991); (b) CP III 40 RS - cimento Portland de alto-forno (NBR 5735, 1991) e cimentos Portland resistentes a sulfatos (NBR 5737, 1992); (c) CP IV 32 - cimento Portland pozolânico (NBR 5736, 1991); e (d) CP V ARI - cimento Portland de alta resistência inicial (NBR 5733, 1991). 
128Revista ALCONPAT, Volumen 4, Número 2, Mayo - Agosto 2014, Páginas 113 - 128

As Tabelas 2 e 3 apresentam os limites da normalização vigente brasileira para as características química, física e mecânica dos cimentos utilizados no presente estudo.

Tabela 2. Propriedades químicas (\% em massa) dos cimentos utilizados - limites de normas.

\begin{tabular}{|c|c|c|c|c|}
\hline Propriedades & CP II-F & CP III RS & CP IV & CP V ARI \\
\hline Clínquer + sulfatos de cálcio & $94-90$ & $40-25$ & $85-45$ & $100-95$ \\
\hline Escória granulada de alto forno & - & $60-70$ & - & - \\
\hline Material pozolânico & - & - & $15-50$ & - \\
\hline Material Carbonático & $6-10$ & $0-5$ & $0-5$ & $0-5$ \\
\hline Óxido de magnésio $(\mathrm{MgO})$ & $\leq 6,5$ & - & 6,5 & 6,5 \\
\hline Trióxido de enxofre $\left(\mathrm{SO}_{3}\right)$ & $\leq 4,0$ & 4,0 & 4,0 & $*$ \\
\hline Perda ao fogo & $\leq 6,5$ & 4,5 & 4,5 & 4,5 \\
\hline Resíduos insolúveis & $\leq 2,5$ & 1,5 & - & 1,0 \\
\hline Anidrido carbônico $\left(\mathrm{CO}_{2}\right)$ & $\leq 5,0$ & 3,0 & 3,0 & 3,0 \\
\hline
\end{tabular}

* $\mathrm{Se}_{3} \mathrm{~A}$ do clínquer $-8 \%, \mathrm{SO}_{3}=3,5 \% ; \mathrm{Se} \mathrm{C}_{3} \mathrm{~A}$ do clínquer $>8 \%, \mathrm{SO}_{3}=4,5 \%$.

Tabela 3. Propriedades físicas e mecânicas dos cimentos utilizados - limites de normas.

\begin{tabular}{|c|c|c|c|c|}
\hline Propriedades & CP II-F & CP III RS & CP IV & CP V ARI \\
\hline Resíduo na peneira $75 \mu \mathrm{m}(\%)$ & $\leq 12$ & $\leq 8$ & $\leq 8$ & $\leq 6$ \\
\hline Área específica $\left(\mathrm{m}^{2} / \mathrm{kg}\right)$ & $\geq 260$ & - & - & $\geq 300$ \\
\hline Início de pega (h) & $\geq 1$ & $\geq 1$ & $\geq 1$ & $\geq 1$ \\
\hline Fim de pega (h) & $\leq 10^{* *}$ & $\leq 12^{* *}$ & $\leq 12^{* *}$ & $\leq 10^{* *}$ \\
\hline Expansibilidade a quente (mm) & $\leq 5$ & $\leq 5$ & $\leq 5$ & $\leq 5$ \\
\hline Expansibilidade a frio (mm) & $\leq 5^{* *}$ & $\leq 5^{* *}$ & $\leq 5^{* *}$ & $\leq 5^{* *}$ \\
\hline Resistência à compressão -3 dias (MPa) & $\geq 10$ & $\geq 12$ & $\geq 10$ & $\geq 24$ \\
\hline Resistência à compressão -7 dias (MPa) & $\geq 20$ & $\geq 23$ & $\geq 20$ & $\geq 34$ \\
\hline Resistência à compressão -28 dias (MPa) & $\geq 32$ & $\geq 40$ & $\geq 32$ & - \\
\hline
\end{tabular}

** Exigências facultativas.

Após a moldagem, os corpos de prova utilizados no ensaio de resistência à compressão permaneceram em câmara úmida (umidade relativa > 95\%) para cura até as datas dos ensaios (7, 28 e 91 dias). As amostras usadas no ensaio de RES ficaram por um período de 28 dias na mesma câmara úmida. Passado esse período, foram realizados os primeiros ensaios de RES. Posteriormente, essas amostras permaneceram em ambiente de laboratório (temperatura $22 \pm 3{ }^{\circ} \mathrm{C}$ e umidade relativa $\pm 65 \%$ ) até a idade de 91 dias, quando foram realizados novos ensaios de resistividade. 
Revista ALCONPAT, Volumen 4, Número 2, Mayo - Agosto 2014, Páginas 113 - 128

A resistência à compressão de cada corpo de prova foi determinada segundo a Norma Brasileira vigente no Brasil (NBR 5739, 2007). Para tal, foi utilizada uma prensa hidráulica, equipada com sistema de pratos com 200 toneladas de capacidade para aplicação de carga.

Em relação ao ensaio de RES, foi executado o método dos quatro pontos. Um equipamento disponível comercialmente foi utilizado para o ensaio. O equipamento possui quatro contatos espaçados a igual distância $(\mathrm{d}=0,05 \mathrm{~m})$. Ao ser posicionado sobre a surpefície do corpo de prova, uma corrente elétrica é aplicada entre os dois contatos externos. A diferença de potencial resultante é medida pelos pelos dois contatos internos. $\mathrm{O}$ equipamento foi calibrado segundo as recomendações do fornecedor antes da realização de cada medida.

\section{RESULTADOS E DISCUSSÕES}

\subsection{Resistência à compressão}

A Fig. 1 apresenta os resultados do ensaio de resistência à compressão realizado nas doze amostras de concreto em 7, 28 e 91 dias.

Os resultados da Fig. 1 comprovam que a resistência à compressão do concreto diminui com o aumento de água na composição do concreto. Esse comportamento também foi identificado por outros autores na literatura (Mehta e Monteiro, 2005; Crauss, 2010; Aït-Mokhtar et al, 2013; Koenders et al, 2014). Alguns autores (Liu et al, 2013) observaram que esse comportamento também é válido para ensaios em pastas de cimento.

Conforme aumenta a idade das amostras de concreto, foi verificado um acréscimo na resistência à compressão. Para a amostra de concreto com cimento CP III 40 RS e relação a/c 0,4, por exemplo, a resistência aos 91 dias de idade foi 52,4\% maior que a resistência do mesmo concreto aos 7 dias. A Fig. 1 também indica que os tipos de cimentos tem influência na resistência do concreto. As amostras compostas por cimentos com maiores teores de adições (isto é, CP IV 32 e CP III 40 RS) apresentaram menores valores de resistência à compressão. Esses resultados estão de acordo com outros estudos (Khatib e Hibbert, 2005; Oner e Akyuz, 2007; Lübeck et al, 2012). No entanto, o diferencial do presente artigo é que a única variação entre as amostras é o tipo de cimento, o que permitiu verificar a influência direta das adições na resistência mecânica dos concretos, sem a interferência de outras variáveis, como os aditivos químicos, por exemplo.

Observa-se também que para as amostras com relação água/cimento de 0,5 e 0,6 e idades de 7, 28 e 91 dias, o cimento CP V ARI apresentou maiores valores de resistência à compressão do que os demais cimentos. Entretanto, o cimento CP II-F 32 superou a resistência do cimento CP V ARI para a condição: a/c=0,40 e 91 dias de idade. Ao se secar o concreto, a quantidade de água que não evapora - isto é, a água que reagiu com os compostos cimentícios e formou os produtos de hidratação - é cerca de $\mathrm{a} / \mathrm{c} \approx 0,23$ (Taylor, 1990). Porém, a relação água/cimento crítica, que permite que o cimento seja completamente hidratado, está entre $0,42-0,44$. Isso ocorre porque a água também apresenta função física na reação de hidratação do cimento, originando espaço entre os grãos para que os produtos da hidratação possam se formar (Klein, 2012). Pode-se observar na Tabela 2 que o cimento CPV ARI possui menor teor de adições do que o CP II-F 32, ou seja, é mais puro e possui mais clínquer. Provavelmente por isso, o CP V ARI necessite de maior quantidade de água para dispersar os grãos e garantir a adequada reação de hidratação. Tal fato pode ter levado a maior resistência à compressão do CP II-F 32 aos 91 dias e a/c=0,40. Esse tipo de cimento, por ter menos clínquer, pode necessitar de menor quantidade de água para adequada dispersão e hidratação. De maneira geral, é provável que a resistência à compressão cresce à medida que são utilizados os seguintes tipos de cimento: CP IV 32, CP III 40 RS, CP II-F 32 e CP V ARI. 
128Revista ALCONPAT, Volumen 4, Número 2, Mayo - Agosto 2014, Páginas 113 - 128

Apesar do cimento CP IV 32 apresentar os menores valores absolutos, o ganho de resistência à compressão (percentual em relação à idade de 7 dias) no tempo foi maior do que o aumento obtido pelas outras amostras. A Fig. 2 apresenta o crescimento percentual da resistência mecânica das amostras com o tempo.
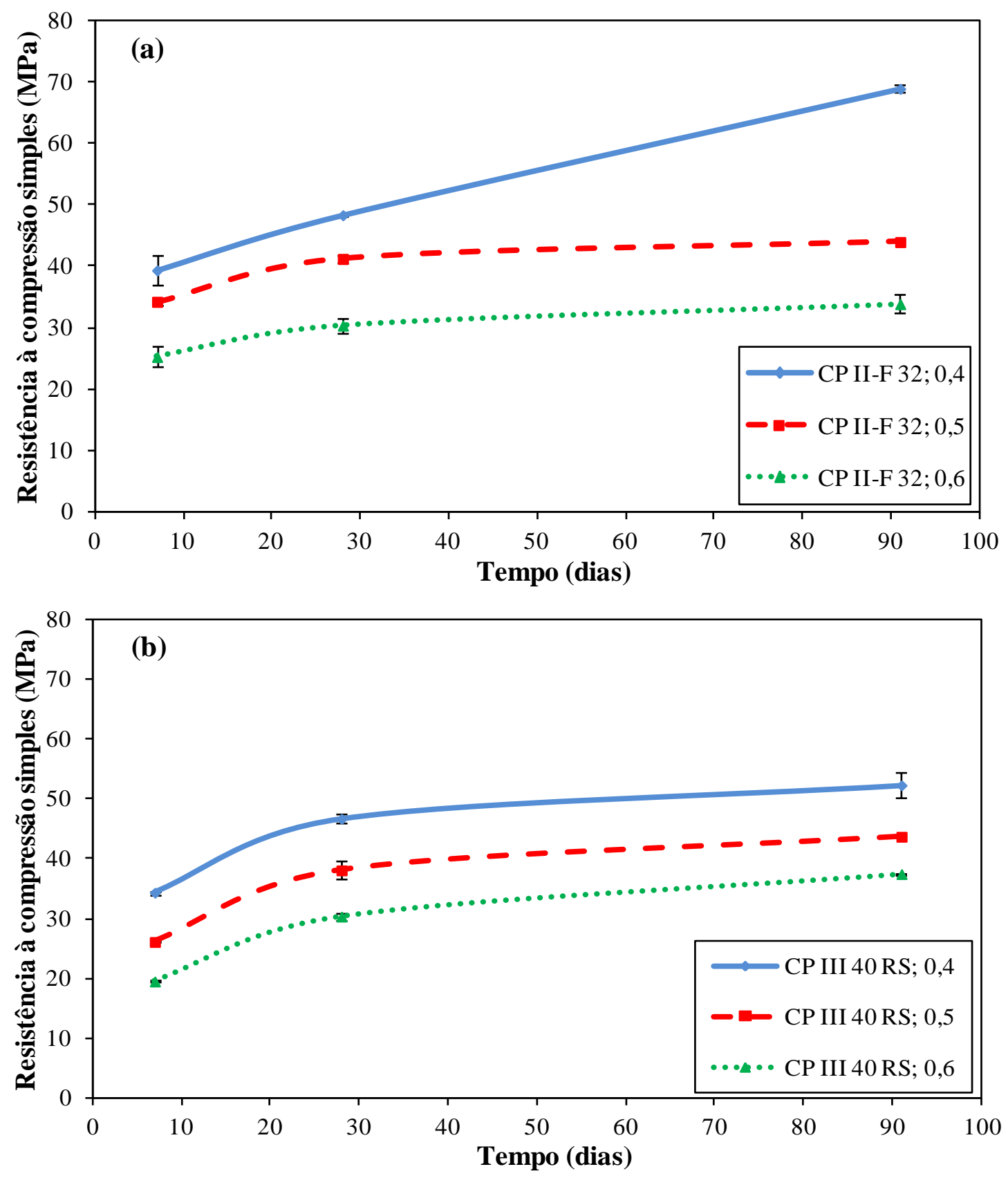

Investigação da resistência à compressão e da resistividade elétrica de concretos com... 
Revista ALCONPAT, Volumen 4, Número 2, Mayo - Agosto 2014, Páginas 113 - 128
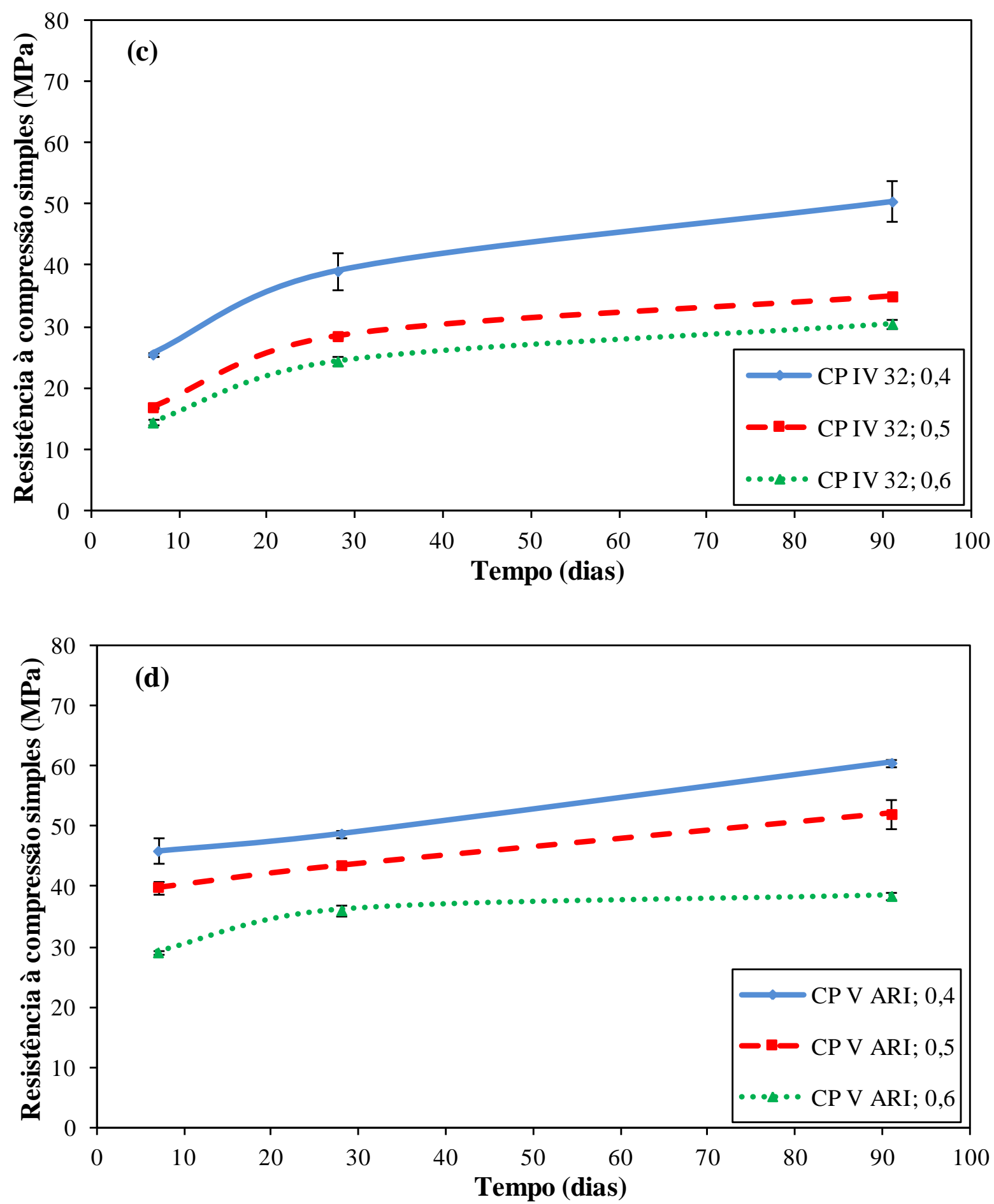

Figura 1. Resultados do ensaio de resistência à compressão: a) CP II-F 32; b) CP III 40 RS; c) CP IV 32; e d) CP V ARI. 


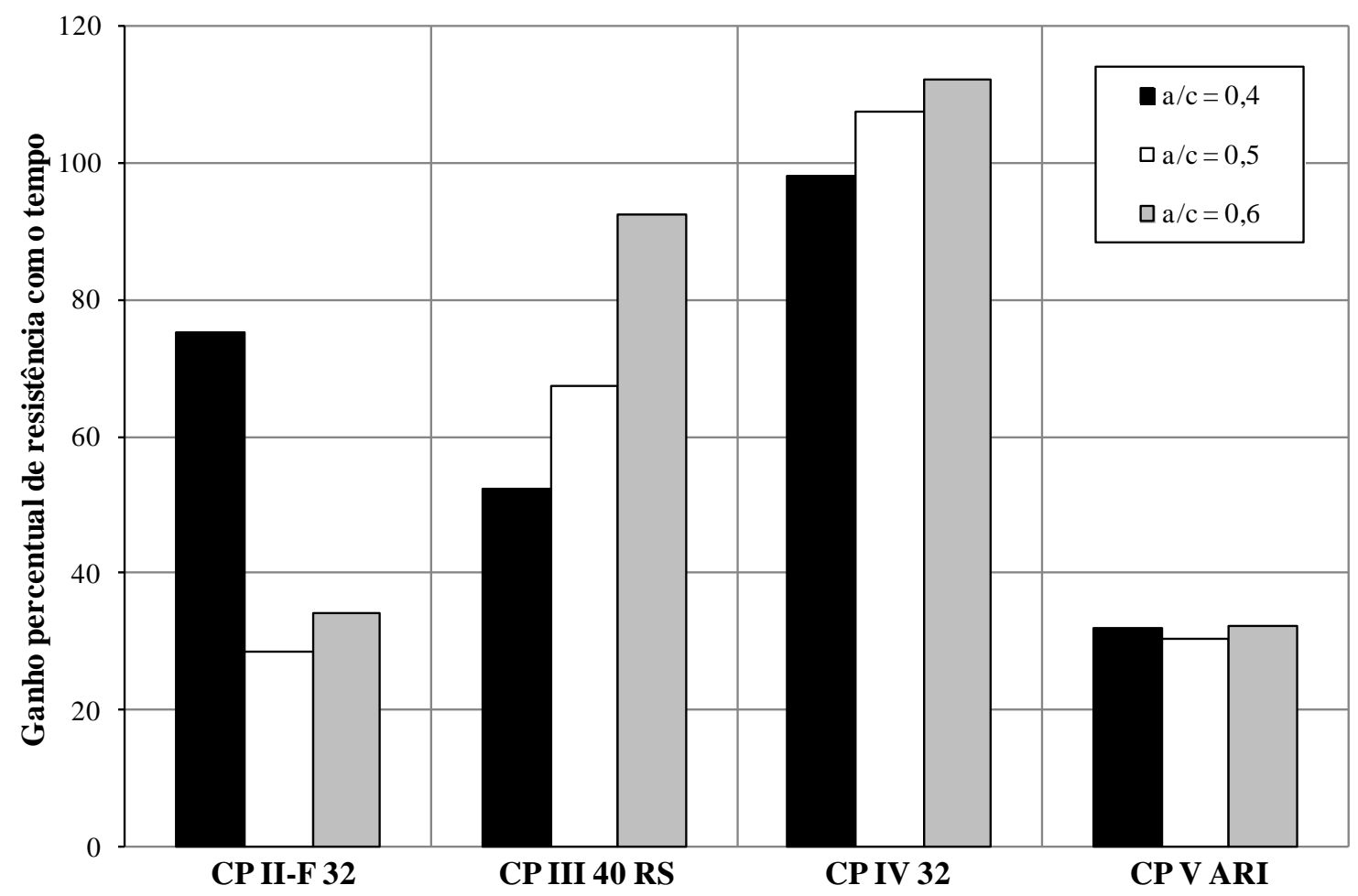

Figura 2. Variação percentual da resistência à compressão obtida de 7 para 91 dias.

Conforme a Fig. 2, as amostras com cimento CP IV 32 chegaram a obter variação na resistência à compressão de mais de 100\%, ao comparar os resultados de $91 \mathrm{com} 7$ dias. Esse elevado ganho percentual se dá pois as reações de hidratação das adições são mais lentas do que as reações dos compostos do clínquer, porém progressivas. As amostras com cimento CP V ARI, por outro lado, apresentam elevada resistência inicial. Essa é uma característica do cimento CP V ARI e está relacionada com a elevada quantidade de clínquer presente na composição química do material (95 a 100\%), como indica a Tabela 2. Apesar da alta resistência inicial, a Fig. 2 mostra que o cimento CP V ARI apresenta pouco ganho percentual de resistência com o tempo quando confrontadas com as demais amostras.

A Fig. 2 também mostra o ganho considerável (cerca de 75\%) da amostra com cimento CP II-F 32 e relação a/c de 0,4 . Conforme comentado antes, essa foi a amostra que apresentou maior valor absoluto de resistência à compressão. 
Revista ALCONPAT, Volumen 4, Número 2, Mayo - Agosto 2014, Páginas 113 - 128

\subsection{Resistividade Elétrica Superficial (RES)}

Foram determinadas a RES das amostras para as idades de 28 e 91 dias. Os resultados podem ser verificados na Fig. 3.
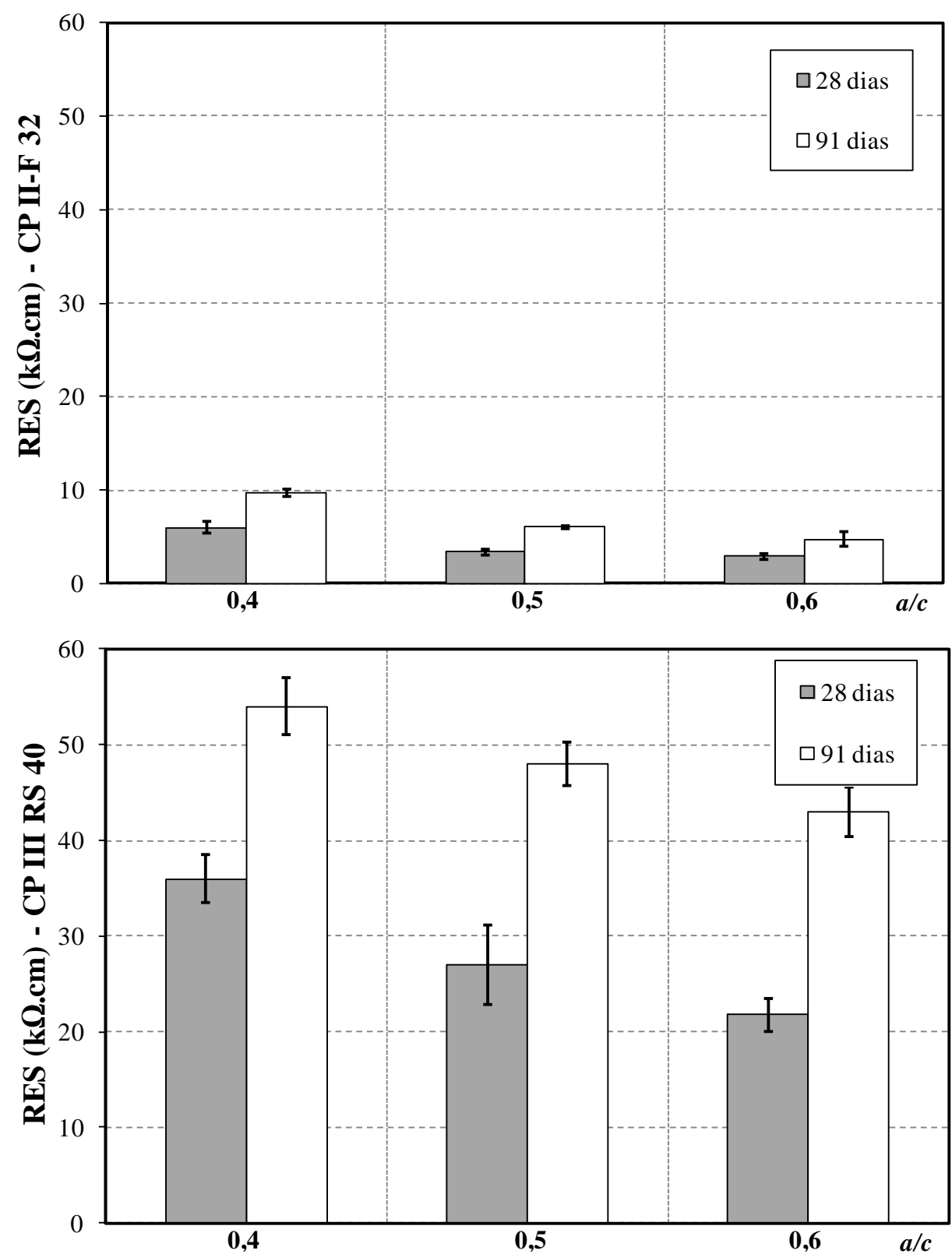
128Revista ALCONPAT, Volumen 4, Número 2, Mayo - Agosto 2014, Páginas 113 - 128
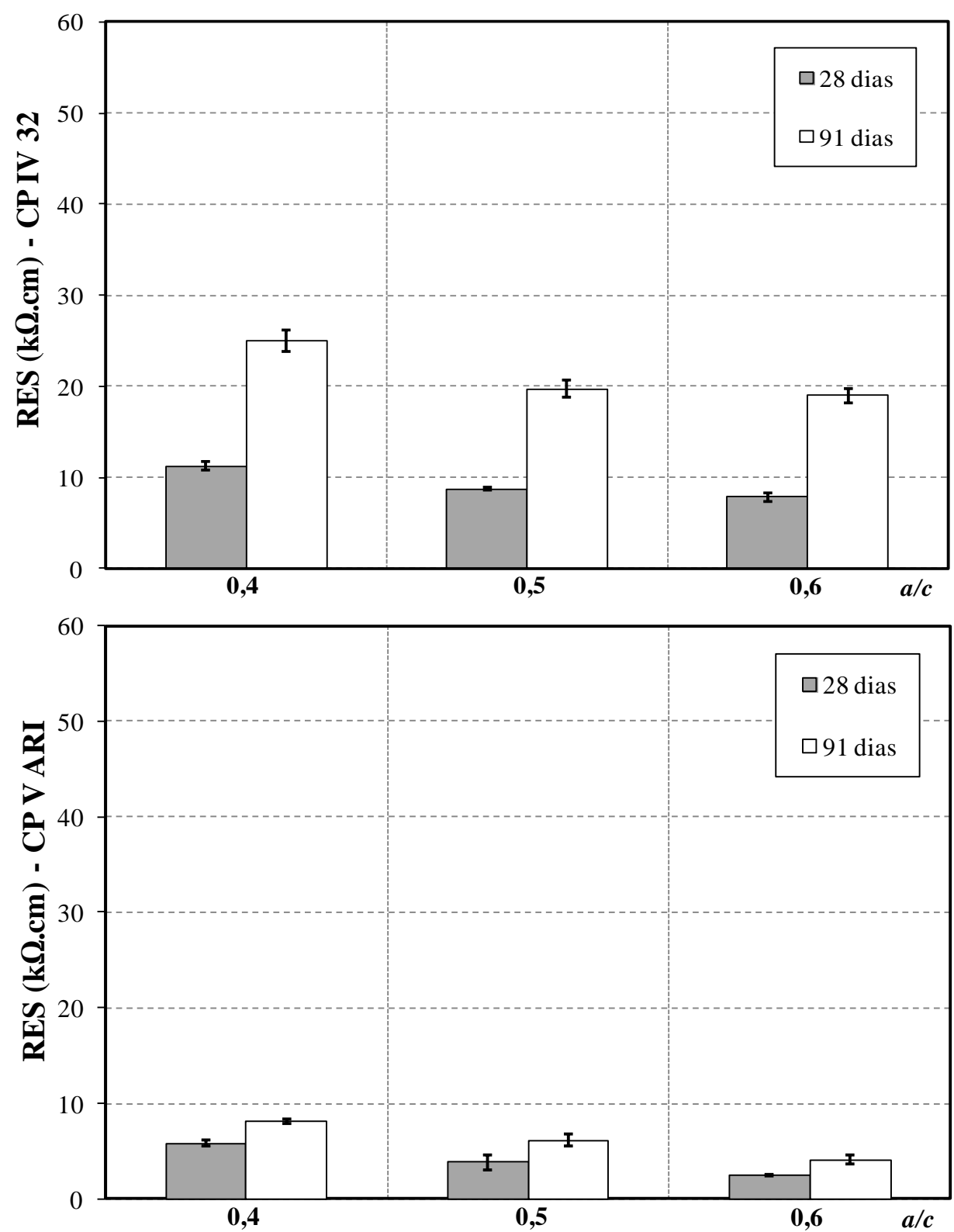

Figura 3. Resultados do ensaio de resistividade elétrica superficial.

De acordo com a Fig. 3, foi identificado um acréscimo da RES com o tempo de ensaio para todas as amostras estudadas, indicando que essa variável tende a crescer no tempo devido à hidratação do cimento e o endurecimento progressivo do concreto. Esse comportamento está de acordo com outros estudos (Andrade e D'Andrea, 2011; Presuel-Moreno et al, 2013). Esses autores verificaram que nas primeiras horas de idade, o concreto apresenta valores mais baixos de RES principalmente devido à formação de uma pasta em suspensão após a mistura do cimento com a água.

Outros autores (Baweja et al, 1997; CCAA, 2009) também identificaram o aumento da RES do concreto no tempo, mas chamam a atenção para um efeito paralelo de redução desse parâmetro com o aumento do ingresso de cloretos no concreto. Além disso, a presença de sulfato ( $\left.\mathrm{SO}_{4}^{--}\right)$na matriz do cimento também contribui para reduzir esse valor (Saleem et al, 1996).

Ao analisar um mesmo tipo de cimento e mesma idade de ensaio, é possível observar que a RES decresce à medida que a relação $\mathrm{a} / \mathrm{c}$ aumenta (Fig. 3). Esse comportamento pode ser explicado pelo fato da solução de poro na estrutura interna do concreto funcionar como um condutor. Sendo

Investigação da resistência à compressão e da resistividade elétrica de concretos com... 
Revista ALCONPAT, Volumen 4, Número 2, Mayo - Agosto 2014, Páginas 113 - 128

assim, quanto maior a quantidade de água nas amostras - combinado com a maior permeabilidade do meio, ambos provenientes da maior relação água/cimento, tem-se uma maior condutividade no interior do concreto, resultando em menores valores de RES. Esses resultados estão em acordo com outros trabalhos (Olsson et al, 2013).

Conforme a Fig. 3, observa-se que a RES para os concretos com cimento CP III 40 RS foram superiores a das outras amostras. Tal comportamento pode ser atribuído à quantidade considerável de adições de escórias de alto forno na composição do cimento CP III 40 RS - cerca de 60-70\% (Tabela 2). Essas adições contribuem para refinar os poros da microestrutura do concreto, resultando em menor condutividade das amostras, o que consequentemente ocasiona em maiores valores de RES.

De acordo com a literatura (Liu et al, 2013), sabe-se que há uma relação inversa entre a porosidade capilar e a resistividade relativa - razão entre a resistividade do material pela resistividade da solução dos poros $\left(\rho_{t} / \rho_{o}\right)$ - em amostras de pastas de cimento. Essa relação é alterada com o grau de hidratação do cimento. Segundo os resultados desses autores, quanto menor a porosidade capilar, maior a resistividade relativa das amostras. Outros estudos (Gesoğlu e Özbay, 2007) mediram valores mais elevados de RES em amostras contendo escórias, ao comparar com concreto sem nenhuma adição.

Colaborando com as afirmações anteriores, segundo os resultados da Fig. 3, as amostras com cimento CP IV 32 foram as que obtiveram segundo melhor desempenho em termos de RES, comportamento explicado pela quantidade significativa de adições pozolônicas (15-50\%) na composição do cimento (Tabela 2). Já os cimentos CP II-F 32 e CP V ARI apresentaram valores inferiores as demais amostras.

As Figs. 4 e 5 apresentam as correlações encontradas entre os resultados de resistência à compressão.

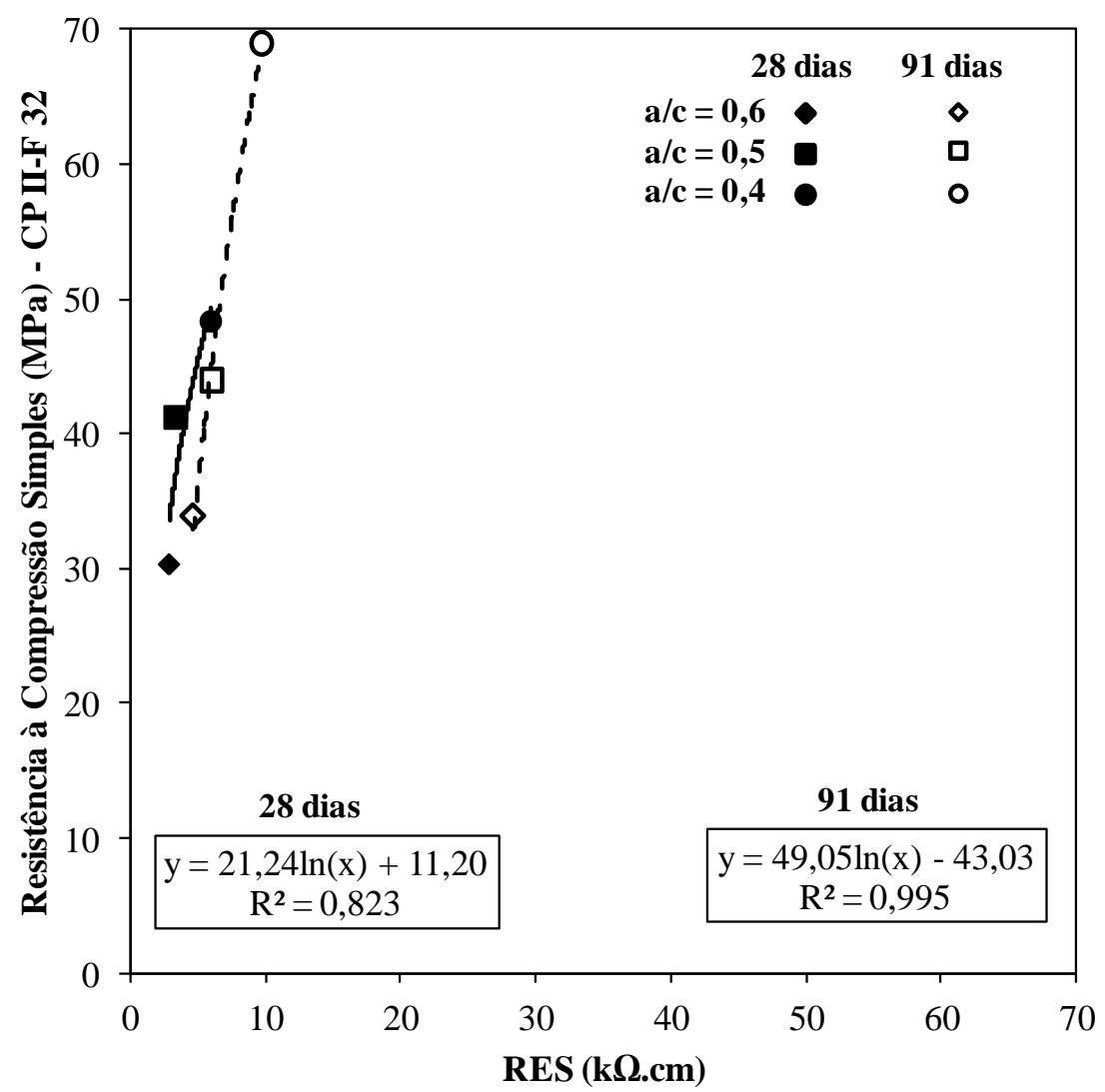

R. A. Medeiros-Junior, M. G. Lima, M. H. F. Medeiros, L. V. Real 
128Revista ALCONPAT, Volumen 4, Número 2, Mayo - Agosto 2014, Páginas 113 - 128

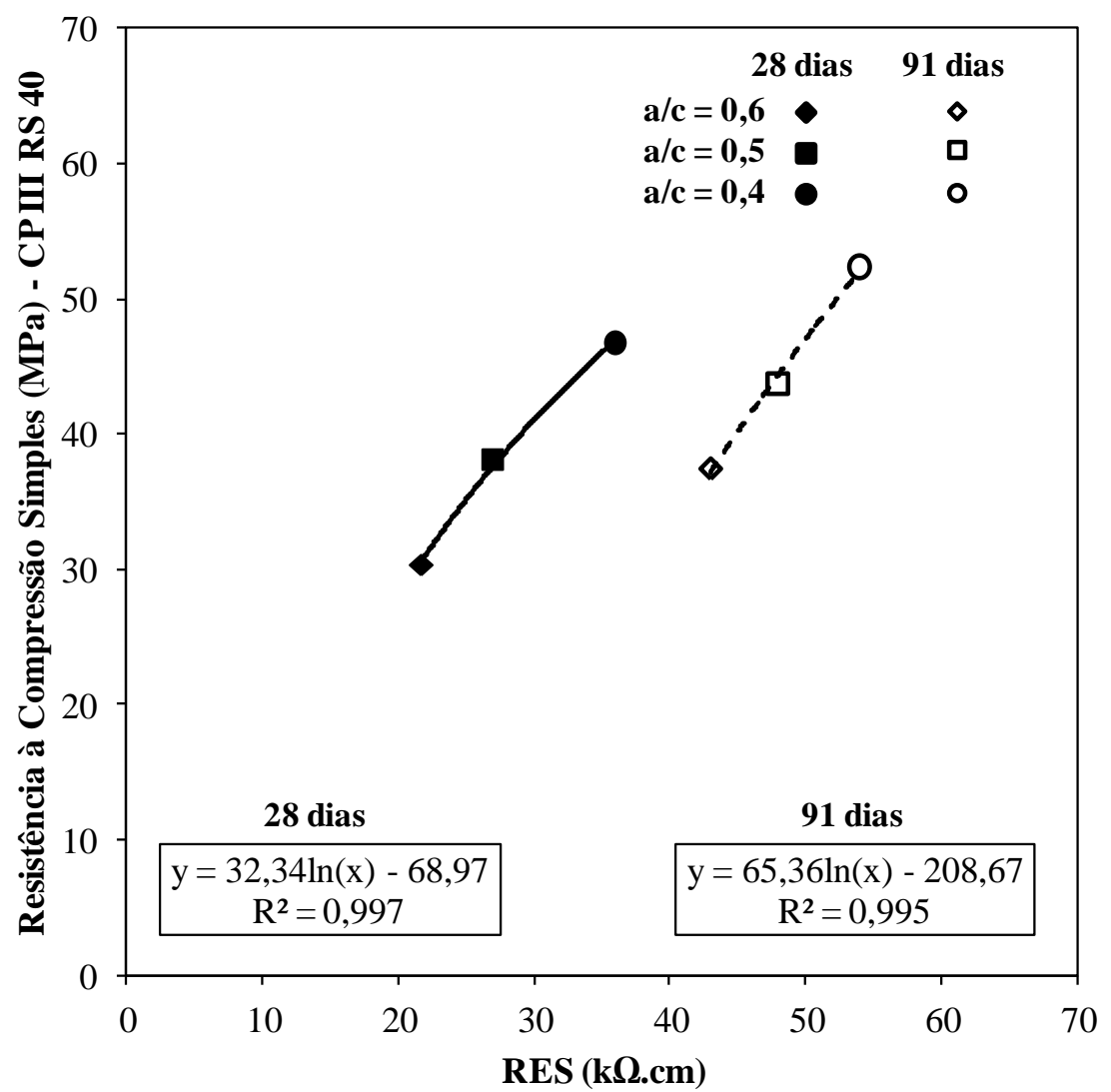

Figura 4. Correlação: resistência à compressão x resistividade elétrica - CP II-F 32 e CP III RS 40.

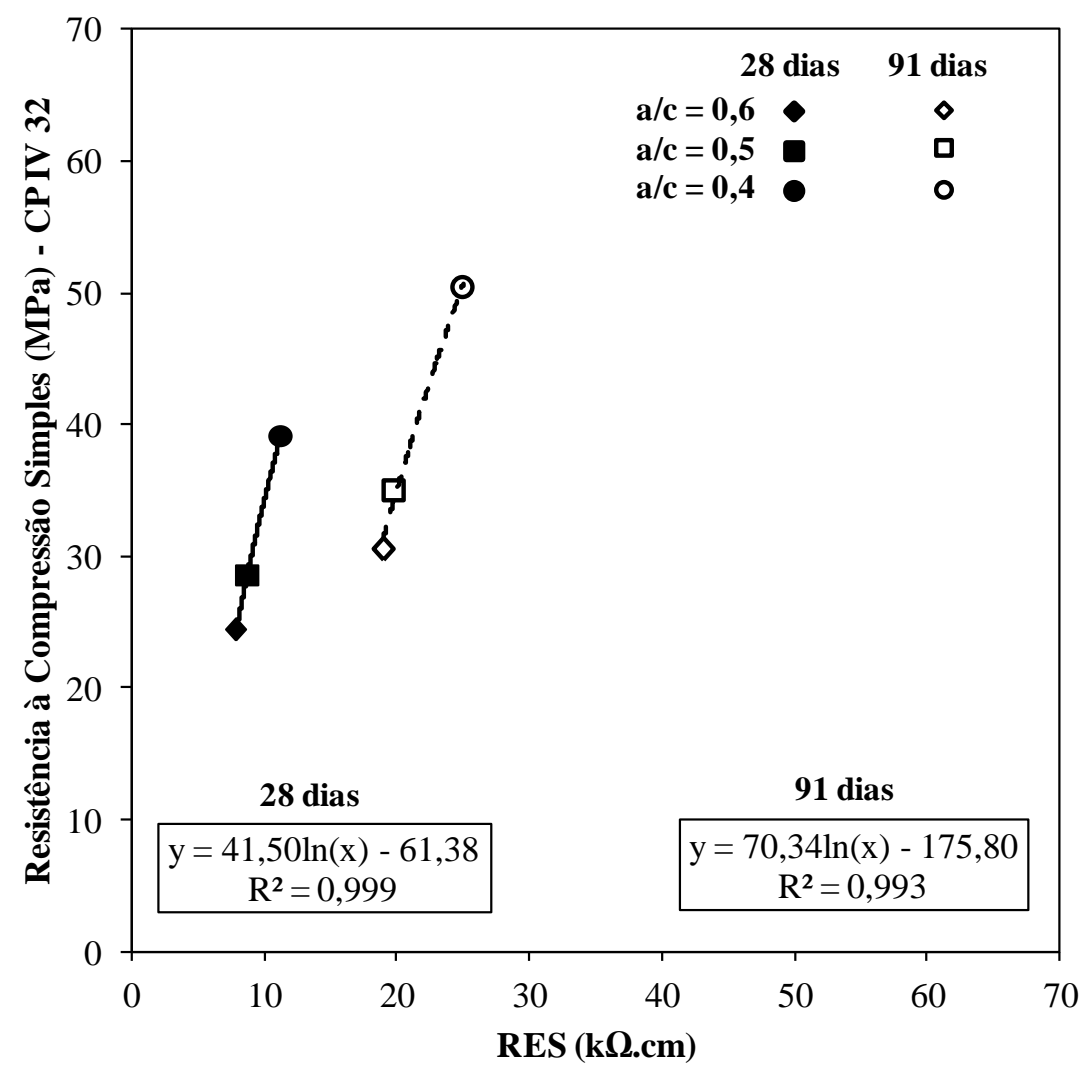

Investigação da resistência à compressão e da resistividade elétrica de concretos com... 
Revista ALCONPAT, Volumen 4, Número 2, Mayo - Agosto 2014, Páginas 113 - 128

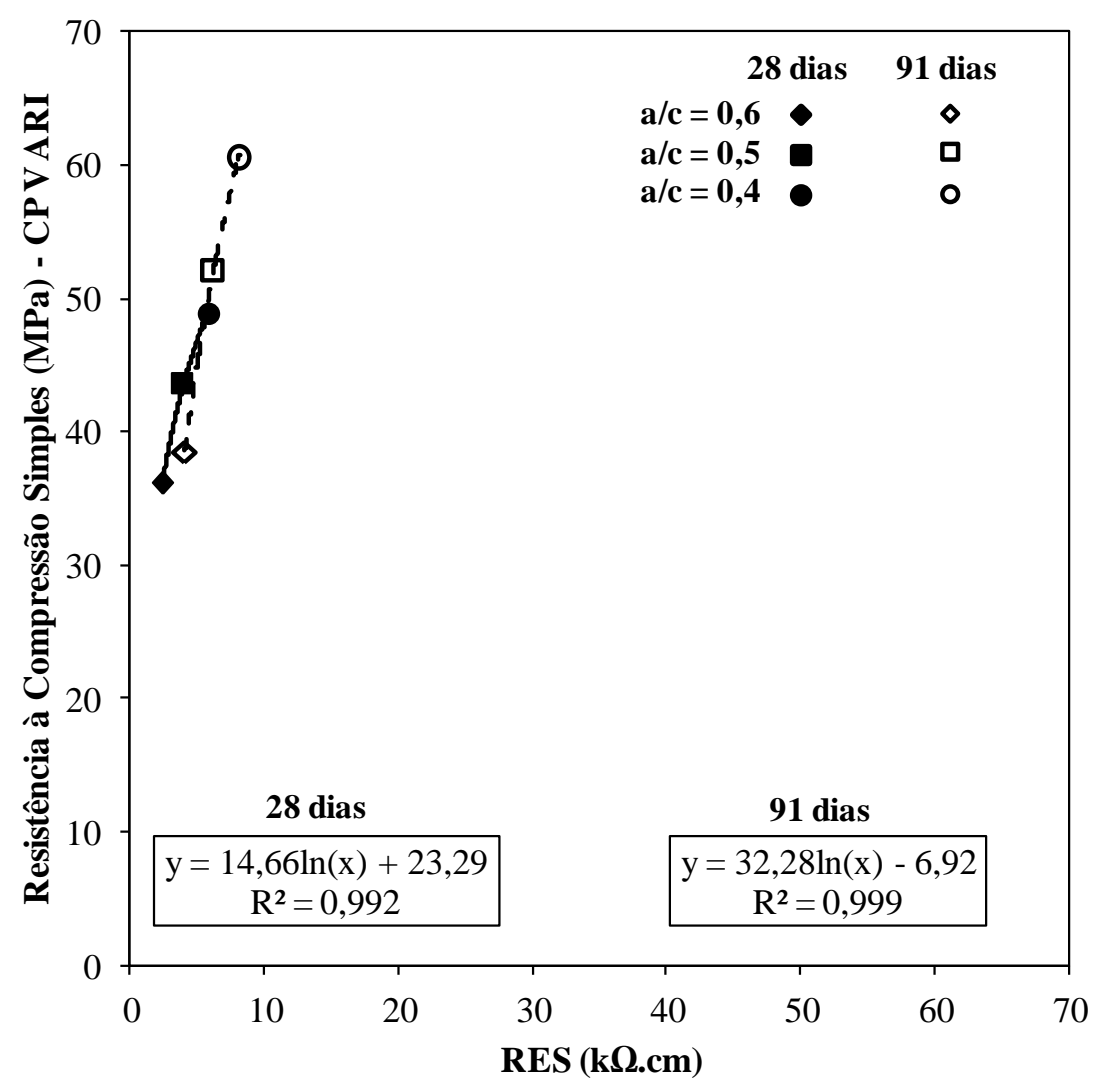

Figura 5. Correlação: resistência à compressão x resistividade elétrica - CP IV 32 e CP V ARI.

Conforme as Figs. 4 e 5, verifica-se que existe uma correlação entre a resistência mecânica e a RES do concreto, que é alterada com o tipo de cimento e com a idade do ensaio. Além disso, para todas as amostras, a curva de correlação que mais se ajustou aos dados foi do tipo logarítmica, com $\mathrm{R}^{2}$ bastante próximos a 1 . Porém, deve-se ressaltar que o número de amostras para determinação dessas correlações não é amplo, o que dificulta realizar afirmações mais precisas a respeito do comportamento dos dados.

Outros estudos (Andrade e D'Andrea, 2011) também encontraram um ajuste logarítmico para correlações entre a resistência mecânica e a RES de uma amostra de concreto. No entanto, não são mencionadas as características do concreto ensaiado no estudo citado.

De acordo com as Figs. 4 e 5, a RES das amostras cresce à medida que a resistência mecânica aumenta, conforme também observado na literatura (Dinakar et al, 2007; Lübeck et al, 2012). Isso significa que com o aumento da densidade da matriz do cimento, devido ao progresso da hidratação, ambos, resistência mecânica e elétrica, tendem a crescer para todas as amostras de cimento verificadas.

\section{CONCLUSÕES}

1. De uma maneira geral, mantendo todos os outros parâmetros do concreto constantes, a resistência do concreto cresce à medida que são utilizados os seguintes tipos de cimento: CP IV 32, CP III 40 RS, CP II-F 32 e CP V ARI. Ressalta-se que particularmente para relação a/c de 0,4 e idade de ensaio de 91 dias, o cimento CP II-F apresentou maior resistência que o cimento CP V ARI. 
128Revista ALCONPAT, Volumen 4, Número 2, Mayo - Agosto 2014, Páginas 113 - 128

2. O cimento CP IV 32 mostrou um maior ganho de resistência (percentual em relação à idade de 7 dias) com o tempo dentre as demais amostras estudadas. Esse comportamento é atribuído a reações de hidratação mais lentas, porém progressivas, ocasionadas pela presença de adições pozolânicas no concreto.

3. Foi observado que a RES aumenta no tempo e com a redução da relação a/c para todos os tipos de cimentos analisados. Os maiores valores de RES foram encontrados para as amostras com cimento CP III 40 RS, com alto teor de escória de alto forno. Essa adição contribui para refinar os poros do concreto, reduzindo a condutividade do meio, e proporcionando maiores valores medidos de RES.

4. A resistência à compressão e a RES das amostras crescem com a idade do concreto. Foi encontrada uma correlação do tipo logarítimica para essas variáveis, com $\mathrm{R}^{2}$ próximos a 1 em todos os casos que fizeram parte deste estudo.

\section{AGRADECIMENTOS}

Os autores agradecem a Fundação de Amparo à Pesquisa do Estado de São Paulo (FAPESP) por fornecer apoio financeiro na elaboração deste estudo e ao Instituto Tecnológico de Aeronáutica (ITA) por providenciar a infraestrutura necessária.

\section{REFERÊNCIAS}

Aït-Mokhtar, A., Belarbi, R., Benboudjema, F., Burlion, N., Capra, B., Carcassès, M., Colliat, J.B., Cussigh, F., Deby, F., Jacquemot, F., Larrard, T. de, Lataste, J.-F., Bescop, P. Le, Pierre, M., Poyet, S., Rougeau, P., Rougelot, T., Sellier, A., Séménadisse, J., Torrenti, J. -M., Trabelsi, A., Turcry, P., Yanez-Godoy, H. (2013), "Experimental investigation of the variability of concrete durability properties", Cement and Concrete Research, V.45, pp. 21-36.

Alonso, C., Andrade C., González, J. A. (1988), "Relation between concrete resistivity and corrosion rate of the reinforcements in carbonated mortar made with several cement types", Cement and Concrete Research, V.18, No. 5, pp. 687-698.

Andrade, C. (2004), "Calculation of initiation and propagation periods of service-life of reinforcements by using the electrical resistivity" in: International Symposium on Advances in Concrete through Science and Engineering, RILEM Symposium. Evanston: Illinois (USA).

Andrade, C., D’Andréa, R. (2011), “La resistividad eléctrica como parámetro de control del hormigón y de su durabilidad”, Revista ALCONPAT, V.1, No. 2, pp. 93-101.

Andrade, C., González, J. A., Feliú, S., Rodriguez, P., Ramírez, E., Alonso, C. (1996), "Some questions on the corrosion of steel in concrete - Part 1: When, how and how much steel corrodes", Materials and Structures, V.29, No. 1, pp. 40-46.

Baweja, D., Roper, H., Sirivivatnanon, V. (1997), "Quantitative descriptions of steel corrosion in concrete using resistivity and anodic polarisation data" in: V. M. Malhotra (Ed.), Proceedings 4th CANMET/ACI International Conference on Durability of Concrete, SP 170-3: pp. 41-63.

CCAA - Cement Concrete \& Aggregates Australia. (2009), "Chloride Resistance of Concrete", Report, June.

Crauss, C. (2010), "Penetração de cloretos em concretos com diferentes tipos de cimento submetidos a tratamento superficial”, Dissertação de Mestrado, Universidade Federal de Santa Maria - UFSM, Santa Maria, Rio Grande do Sul: p. 100.

Dinakar, P., Babu, K. G., Santhanam, M. (2007), "Corrosion behavior of blended cements in low and medium strength concretes", Cement and Concrete Composites, V.29, No. 2, pp. 136-145. 
Revista ALCONPAT, Volumen 4, Número 2, Mayo - Agosto 2014, Páginas 113 - 128

Gayarre, F. L., Pérez, C. L., López, M. A. S., Cabo, A. D. (2014), "The effect of curing conditions on the compressive strength of recycled aggregate concrete", Construction and Building Materials, V.53, pp. 260-266.

Gesoğlu, M., Özbay, E. (2007), "Effects of mineral admixtures on fresh and hardened properties of self-compacting concretes: binary, ternary and quaternary systems", Materials and Structures, V.40, No. 9, pp. 923-937.

Ghods, P.; Isgor, O. B., Pour-Ghaz, M. (2007), "A practical method for calculating the corrosion rate of uniformly depassivated reinforcing bars in concrete", Materials and Corrosion, V.58, No. 4, pp. 265-272.

Gowers, K. R., Millard, S. G. (1999), "Measurement of concrete resistivity for assessment of corrosion severity of steel using wenner technique”, ACI Materials Journal, V.96, M66, pp. 536541.

Gulikers, J. (2005), "Theoretical considerations on the supposed linear relationship between concrete resistivity and corrosion rate of steel reinforcement", Materials and Corrosion, V.56, No. 6, pp. 393-403.

Halliday, D., Resnick, R. (1994), Fundamentos da física. Eletromagnetismo. 3.ed., v. 3, (Rio de Janeiro: LCT).

Helene, P. (2011), “Análise da resistência à compressão do concreto em estruturas acabadas com vistas à revisão da segurança estrutural”, Revista ALCONPAT, V.1, No. 1, pp. 67-92.

Khatib, J., Hibbert, J. J. (2005), "Selected engineering properties of concrete incorporating slag and metakaolin", Construction and Building Materials, V.19, No. 6, pp. 460-472.

Klein, N. S. (2012), "El rol físico del água em mezclas de cemento Portland”, Tese de Doutorado, Universitat Politècnica de Catalunya, Barcelona.

Koenders, E. A. B., Pepe, M., Martinelli, E. (2014), "Compressive strength and hydration processes of concrete with recycled aggregates", Cement and Concrete Research, V.56, pp. 203212.

Liu, Z., Zhang, Y., Liu, L., Jiang, Q. (2013), “An analytical model for determining the relative electrical resistivity of cement paste and $\mathrm{C}-\mathrm{S}-\mathrm{H}$ gel", Construction and Building Materials, V.48, pp. 647-655.

Lübeck, A., Gastaldini, A. L. G., Barin, D. S., Siqueira, H. C. (2012), “Compressive strength and electrical properties of concrete with white Portland cement and blast-furnace slag", Cement and Concrete Composites, V.34, No. 3, pp. 392-399.

Medeiros, M. H. F., Pereira, E., Helene, P. (2012), "Tratamento de superfície com silicato de sódio para concreto: penetração de cloretos e absorção de água”, Revista ALCONPAT, V.2, No. 3, pp. 157-169.

Mehta, P. K., Monteiro, P. J. M. (2005), Concrete: microstructure, properties and materials. $3^{\mathrm{a}}$ Ed., (McGraw-Hill Professional).

NBR 5733 (1991), "Cimento Portland de alta resistência inicial. Associação Brasileira de Normas Técnicas" - ABNT. Rio de Janeiro, Brasil.

NBR 5735 (1991), "Cimento Portland de alto-forno. Associação Brasileira de Normas Técnicas" - ABNT. Rio de Janeiro, Brasil.

NBR 5736 (1991), “Cimento Portland pozolânico. Associação Brasileira de Normas Técnicas”ABNT. Rio de Janeiro, Brasil.

NBR 5737 (1992), "Cimentos Portland resistentes a sulfatos. Associação Brasileira de Normas Técnicas" - ABNT. Rio de Janeiro, Brasil.

NBR 5738 (2003), "Concreto - Procedimento para moldagem e cura de corpos-de-prova. Associação Brasileira de Normas Técnicas" - ABNT. Rio de Janeiro, Brasil.

NBR 5739 (2007), "Concreto - Ensaios de compressão de corpos-de-prova cilíndricos. Associação Brasileira de Normas Técnicas” - ABNT. Rio de Janeiro, Brasil.

R. A. Medeiros-Junior, M. G. Lima, M. H. F. Medeiros, L. V. Real 
128Revista ALCONPAT, Volumen 4, Número 2, Mayo - Agosto 2014, Páginas 113 - 128

NBR 11578 (1991), “Cimento Portland composto - Especificação. Associação Brasileira de Normas Técnicas" - ABNT. Rio de Janeiro, Brasil.

NBR 12655 (2006), "Concreto de cimento Portland - Preparo, controle e recebimento Procedimento. Associação Brasileira de Normas Técnicas" - ABNT. Rio de Janeiro, Brasil.

Olsson, N.; Baroghel-Bouny, V.; Nilsson, L.; Thiery, M. (2013), "Non-saturated ion diffusion in concrete - A new approach to evaluate conductivity measurements", Cement and Concrete Composites, V.40, pp. 40-47.

Oner, A. E., Akyuz, S. (2007), "An experimental study on optimum usage of GGBS for the compressive strength of concrete", Cement and Concrete Composites, V.29, No. 6, pp. 505-514.

Pereira, V. G. A. (2001), "Avaliação do coeficiente de difusão de cloretos em concretos: influência do tipo de cimento, da relação a/c, da temperatura e do tempo de cura”, Dissertação de Mestrado, Universidade Federal do Rio Grande do Sul - UFRGS, Porto Alegre, Rio Grande do Sul, p. 154.

Presuel-Moreno, F., Wu, Y.-Y., Liu, Y. (2013), "Effect of curing regime on concrete resistivity and aging factor over time”, Construction and Building Materials, V.48, pp. 874-882.

Saleem, M., Shameemt, M., Hussain, S. E., Maslehuddin, M. (1996), "Effect of moisture, chloride and sulphate contamination on the electrical resistivity Portland cement concrete", Construction and Building Materials, V.10, No. 3, pp. 209-214.

Santor, M. S., Gastaldini, A. L. G., Crauss, C., dos Santos, G. T., Rossini, F. C. (2012), "Influência de materiais de proteção na resistividade elétrica do concreto", Revista ALCONPAT, V.2, No. 1, pp. 47-57.

Silva, B. A. (2009), "Análise da influência do tipo de cura na resistência à compressão de corpos de prova de concreto", Trabalho de Graduação, Instituto Tecnológico de Aeronáutica - ITA, São José dos Campos, São Paulo, p. 48.

Solís, R. G., Moreno, E. I., Arjona, E. (2012), "Resistencia de concreto con agregado de alta absorción y baja relación a/c”, Revista ALCONPAT, V.2, No. 1, pp. 21-29.

Taylor, H. F. W. (1990), Cement chemistry (London: Academic Press).

Tessari, R. (2001), "Estudo da capacidade de proteção de alguns tipos de cimento nacionais, em relação à corrosão de armaduras sob a ação de íons cloreto”, Dissertação de Mestrado, Universidade Federal do Rio Grande do Sul - UFRGS, Porto Alegre, Rio Grande do Sul, p. 114. 